\title{
FÍSTULA CERVICAL PÓS-ANASTOMOSE ESOFAGOGÁSTRICA: É POSSÍVEL DIMINUIR A OCORRÊNCIA?
}

\section{Is it possible to reduce the fistula rate of the cervical esophagogastric anastomosis after oesophagectomy?}

\author{
Paulo Roberto Ott FONTES, Cristine Kist KRUSE, Fábio Luiz WAECHTER, Mauro NECTOUX, \\ Larissa Bittencourt Saggin FOCHESATO, Gustavo Ferreira GOETTERT, \\ Maurício Fraga da SILVA, Luiz PEREIRA-LIMA
}

ABCDDV/617

Fontes PRO, Kruse CK, Waechter FL, Nectoux M, Fochesato LBS, Goettert GF, Silva MF, Pereira-Lima L. Fístula cervical pós-anastomose esofagogástrica: é possível diminuir a ocorrência? ABCD Arq Bras Cir Dig. 2008;21(4):158-63

RESUMO - Racional - O câncer de esôfago é a sexta causa de morte relacionada à neoplasia no Brasil, e a esofagectomia quando factível é um dos pilares do tratamento, tanto com intento curativo quanto paliativo. A fístula cervical é complicação comum do procedimento e tem incidência entre 0,8 e 47, 6\%. Objetivo - Testar a eficiência de uma alternativa técnica para a diminuição desta ocorrência. Métodos - Análise prospectiva de 126 pacientes alocados em dois grupos de acordo com a técnica utilizada para a anastomose esofagogástrica cervical. O grupo A, composto por 96 pacientes, teve a anastomose cervical realizada em dois planos, camadas mucosa com sutura contínua de fio categute cromado 3-0 ou caprofyl 3-0 e seromuscular com pontos separados de seda 3-0 agulhada, de forma tradicional, após ressecção prévia das extremidades redundantes do esôfago e tubo gástrico. $\mathrm{O}$ grupo $\mathrm{B}$, composto por 25 pacientes, foi submetido a uma variação técnica para esta anastomose, inicialmente sem a abertura da camada mucosa da extremidade redundante do tubo gástrico e coto esofágico. Resultados - O grupo no qual foi efetuada a nova alternativa técnica de esofagogastroanastomose cervical apresentou incidência nula de deiscência de sutura cervical. Conclusão - A alternativa técnica proposta foi eficiente no quesito fístula por otimizar a apresentação dos planos de sutura, facilitando a confecção da anastomose, diminuindo assim as taxas desta complicação na esofagectomia

DESCRITORES - Esofagectomia. Neoplasia do esôfago.

\section{INTRODUÇÃO}

A esofagectomia é procedimento cirúrgico complexo e tradicionalmente associado à morbidade significativa, tendo como principal indicação o tratamento da doença maligna. Neste caso, apesar dos progressos em outras modalidades terapêuticas, a esofagectomia persiste como tratamento de escolha em pacientes com tumores potencialmente ressecáveis, tanto com intento curativo quanto paliativo. Entretanto, na afecção maligna, cerca de $75 \%$ dos pacientes já apresentam doença avançada no momento do diagnóstico ${ }^{24,25,59,62}$. O câncer de esôfago figura entre os tumores sólidos mais letais, sendo referido universalmente como a sexta causa de morte dentre as neoplasias. Conforme estimativa do Instituto Nacional do Câncer - INCA, no Brasil são previstos registros de cerca de 10640 casos no ano de 2008.

Atualmente, a seleção apurada de pacientes, associada ao aperfeiçoamento da anestesiologia, do rigor técnico e

Trabalho realizado no Departamento de Cirurgia da Universidade Federal de Ciências da Saúde de Porto Alegre - UFCSPA, Porto Alegre, RS, Brasil.

Correspondência: Paulo Roberto Ott Fontes, e-mail: prfontes@terra.com.br do manejo pré e pós-operatório em unidades de terapia intensiva resultaram em melhora significativa nas taxas de morbi-mortalidade destes pacientes ${ }^{5,46,62}$. Da mesma forma, a literatura evidencia que os bons resultados em procedimentos cirúrgicos complexos como a esofagectomia são volume dependentes ${ }^{11,39,43,57}$, com taxas de mortalidade hospitalar em serviços de excelência inferiores a $5 \%{ }^{62}$.

Após ressecção cirúrgica com intuito curativo, taxas de sobrevida em cinco anos são referidas entre 10 e $40 \%$ na maioria dos serviços de excelência. Já no Japão, Akiyama, entre outros, há muito refere resultados mais alentadores em cinco anos1. Por outro lado, visto o prognóstico reservado em nosso meio, é imperiosa a manutenção da qualidade de vida destes pacientes, concentrando esforços na prevenção de complicações que possam causar morbidade. Dentre estas, a mais temida é a deiscência da anastomose cervical, que é o calcanhar de Aquiles desta operação, com manifestações que oscilam entre a ocorrência benigna de sinus ou fístula até apresentações graves como mediastinite e óbito.

Este estudo tem o objetivo de apresentar nova alternativa técnica de anastomose cervical visando diminuir a ocorrência de deiscência e consequentemente melhorar os resultados finais das esofagectomias 


\section{MÉTODOS}

Análise de pacientes submetidos à esofagectomia no complexo Hospitalar Santa Casa e Universidade Federal de Ciências da Saúde de Porto Alegre entre os anos de 1994 e 2008 por um mesmo grupo. Os dados dos pacientes foram coletados em um protocolo do serviço e os prontuários médicos hospitalares foram revistos quando informações adicionais foram necessárias.

De um total de 625 pacientes registrados entre operados e atendidos, separou-se aleatoriamente 96 para o grupo A, que foi comparado com os 25 consecutivos do grupo B no que se refere ao objetivo de analisar o quesito fístula cervical em relação à técnica cirúrgica da anastomose esofagogástrica cervical. Nos dois grupos, após a esofagectomia, o trânsito alimentar foi reconstituído com tubo gástrico transmediastinal posterior transposto a região cervical (Figura 1). O grupo A teve a anastomose cervical realizada em dois planos, camadas mucosa com sutura contínua de fio categute cromado 3-0 ou caprofyl 3-0 e seromuscular com pontos separados de seda 3-0 agulhada, de forma tradicional no serviço dos autores, após ressecção prévia das extremidades redundantes do esôfago e tubo gástrico. O grupo $\mathrm{B}$ foi submetido à variação técnica para esta anastomose, inicialmente sem a abertura da camada mucosa da extremidade redundante do tubo gástrico e coto esofágico (Figura 2). Assim, após seromiotomia gástrica e miotomia circular nos cotos respectivos foi realizada uma primeira linha de sutura seromuscular posterior (excluída a mucosa). Após a anastomose posterior, efetuou-se a anastomose da mucosa com caprofyl ou categute em sutura contínua (Figura 3). A seguir, foram ressecadas as extremidades redundantes e completada a anastomose seguindo as mesmas linhas da sutura posterior agora na sua face anterior, primeiro a camada mucosa em sutura contínua com mesmo fio e a seguir a seromuscular da mesma forma com pontos separados de seda 3-0 (Figura 4).

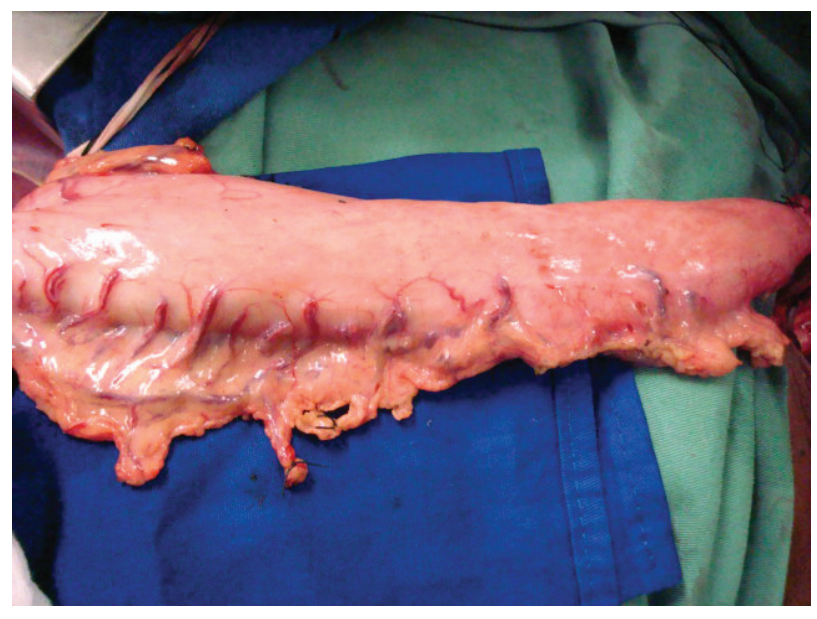

FIGURA 1 - Tubo gástrico transmediastinal para ser transposto

à região cervical

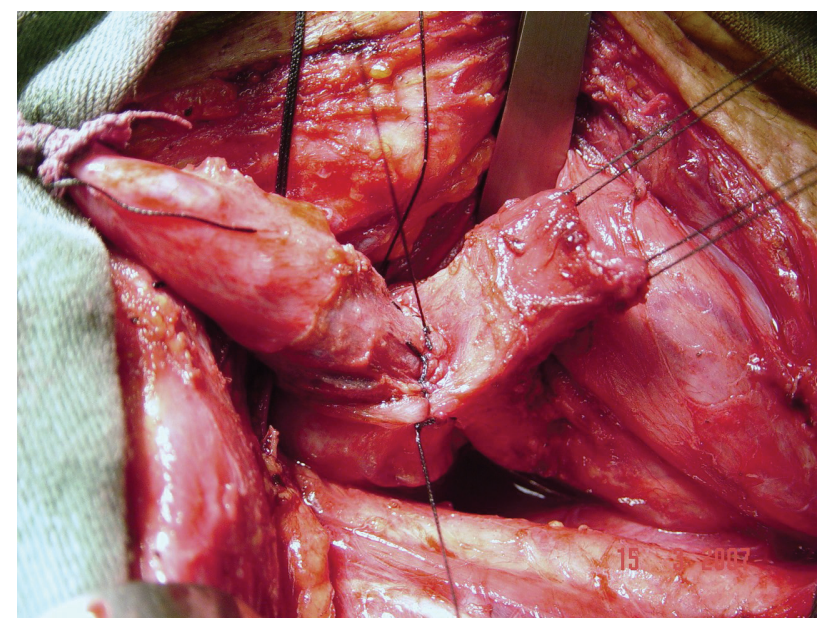

FIGURA 2 - Sutura sem abertura da camada mucosa entre o tubo gástrico e o coto esofágico

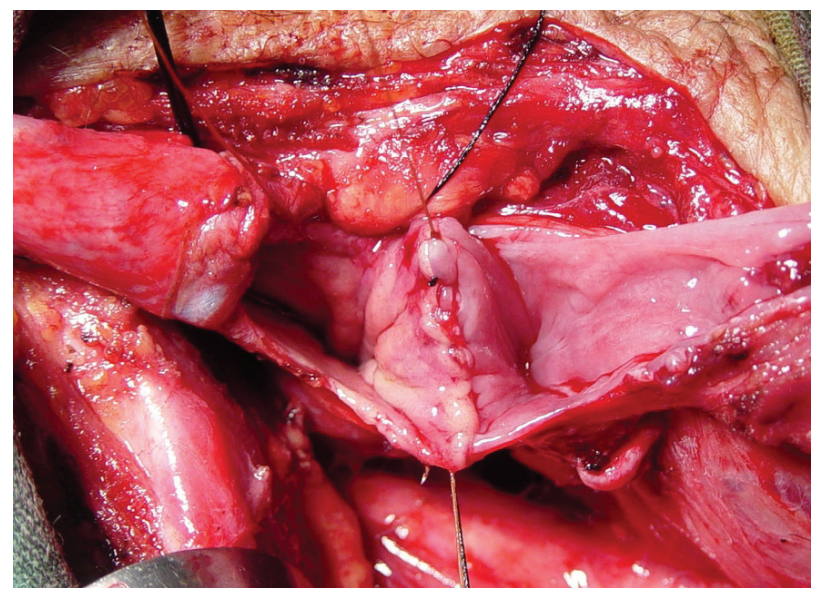

FIGURA 3 - Sutura contínua na anastomose da mucosa esôfagogástrica

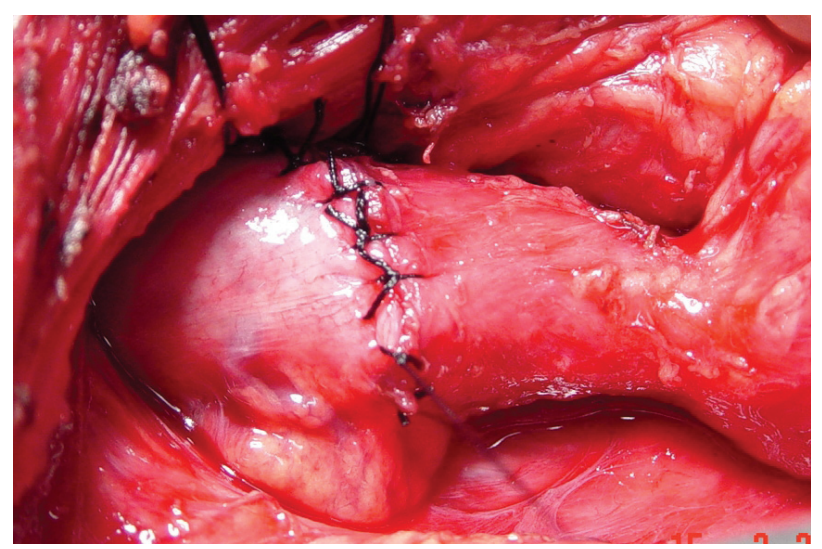

FIGURA 4 - Sutura seromuscular entre o tubo gástrico e o coto esofágico à região cervical 
As características da amostra estão listadas na Tabela 1.

TABELA 1 - Dados dos dois grupos do estudo

\begin{tabular}{|c|c|c|c|}
\hline & Grupo A & Grupo B & $P$ \\
\hline $\mathrm{N}$ & 96 & 25 & \\
\hline $\begin{array}{l}\text { Sexo masculino } \\
(\%)\end{array}$ & 72,3 & 72 & NS \\
\hline Idade média (anos) & $56,80 \pm 8,04$ & $62,48 \pm 10,44$ & $P<0,01$ \\
\hline Uso de álcool (\%) & 51 & 29,2 & NS \\
\hline Tabagismo (\%) & 84,2 & 88 & NS \\
\hline $\begin{array}{l}\text { Emagrecimento > } \\
10 \%(\%)\end{array}$ & 49,5 & 28 & NS \\
\hline Albumina (g/dl) & $3,98 \pm 0,50$ & $3,96 \pm 0,52$ & NS \\
\hline \multicolumn{4}{|l|}{ ASA $(\%)$} \\
\hline I & 0 & 8 & \\
\hline II & 62,4 & 48 & NS \\
\hline III & 37,6 & 49 & \\
\hline \multicolumn{4}{|l|}{ Via: } \\
\hline Toracotomia (\%) & 59,4 & 80 & NS \\
\hline Transhiatal (\%) & 40,6 & 20 & \\
\hline Sangramento (ml) & $409,16 \pm 296,81$ & $467,60 \pm 418,82$ & NS \\
\hline $\begin{array}{l}\text { Vasopressor intra- } \\
\text { operatório (\%) }\end{array}$ & 15,8 & 4 & NS \\
\hline $\begin{array}{l}\text { Vasopressor UTI } \\
(\%)\end{array}$ & 9,9 & 23,1 & NS \\
\hline \multicolumn{4}{|l|}{ Tipo histológico } \\
\hline $\begin{array}{l}\text { - Carcinoma epi- } \\
\text { dermóide }\end{array}$ & 83,2 & 80 & NS \\
\hline - Adenocarcinoma & 16,8 & 20 & \\
\hline $\begin{array}{l}\text { Deiscência anasto- } \\
\text { mose cervical (\%) }\end{array}$ & 18,8 & 0 & $P<0,01$ \\
\hline
\end{tabular}

A análise estatística foi realizada através do programa Epi-info 3.5.1.

\section{RESULTADOS}

Dos 121 pacientes, cinco foram submetidos à esplenectomia por lesão intra-operatória, dois à colecistectomia por colelitíase sintomática, um à lobectomia pulmonar por carcinoma bronquioloalveolar sincrônico, dois à sigmoidectomia por adenocarcinoma, todos no mesmo tempo cirúrgico. Um paciente apresentou situs inversus totalis e cirrose como achado transoperatório.

Os dois grupos foram semelhantes clinicamente conforme testado do ponto de vista estatístico e demonstrado na Tabela 1, excetuado pelos valores de idade média da amostra, que atingiu $P<0,01$. O diagnóstico de deiscência da anastomose cervical foi clínico e, em pacientes assintomáticos, pela realização rotineira de estudo radiológico contrastado de hipofaringe, esôfago cervical e torácico em incidências antero-posterior/perfil/oblíquo no $10^{\circ}$ dia do pós-operatório. Assim, a taxa de deiscência foi de $18,8 \%$ no grupo A e nula no grupo $\mathrm{B}$, com $P<0,01$, apresentando risco relativo de 0,81\% (intervalo de confiança - 95\%: $0,74-0,89$ ).

\section{DISCUSSÃO}

A esofagectomia para tratamento de câncer tem como objetivo principal a intenção de cura ou paliação do câncer com consequente alívio da disfagia ${ }^{45,51,52,61}$. A prevenção das complicações tem se mostrado essencial para o sucesso da esofagectomia ${ }^{4,18,29,38,40,54,55,58}$, visto que seu impacto tem efeito negativo na sobrevida dos mesmos devido ao já prognóstico reservado da neoplasia esofágica per se $\mathrm{e}^{8,25,27,54,58,60}$. Como a deiscência de anastomose esofagogástrica cervical está relacionada ao aumento da morbidade pós-operatória e a efeitos deletérios na qualidade de vida a curto e longo prazo em comparação aos que não desenvolveram tal intercorrência ${ }^{3}, 12,13,27,41,44,48,54,63$, a procura de alternativas técnicas visando minimizar ainda mais esta ocorrência faz-se justificada.

A seleção cuidadosa de pacientes para a operação, visando a redução da morbidade pós-operatória, é atitude enfatizada e preconizada pela literatura médica ${ }^{7,32,33,54,62}$. Assim, os pacientes submetidos às duas alternativas técnicas foram avaliados com ênfase sob o ponto de vista cardiológico, respiratório e nutricional, sendo submetidos à operação apenas os com condições clínicas ideais, visto que o melhor manejo das complicações pós-operatórias é a sua prevenção. Desta forma, a ressecabilidade e a reserva funcional dos pacientes devem ser corretamente avaliadas no pré-operatório, para evitar operações desnecessárias ou de risco ${ }^{29,30,56}$. Valorizam-se os níveis séricos de albumina ${ }^{17}$, sendo excepcional pelos autores a realização da operação em vigência de valores inferiores a $3 \mathrm{~g} / \mathrm{dL}$.

A esofagectomia subtotal com anastomose cervical é o procedimento de escolha para o tratamento da neoplasia esofágica em centros de referência ${ }^{16,19,20,50,51,52}$, sendo realizada em por estes autores em todos os pacientes clinicamente aptos e com doença potencialmente ressecável. Em doentes que não atingem essas condições, a paliação é realizada através de tunelização tumoral por prótese cirúrgica $^{20,23,25,35,36,37}$. Porém, mesmo quando não há intuito curativo, é amplamente aceito que a esofagectomia é a melhor paliação $0^{20,47,50}$, exceto em pacientes com doença disseminada. Aos pacientes com comorbidades que inviabilizem quaisquer procedimentos cirúrgicos, pode ser reservado o restabelecimento de uma via de alimentação através de sondagem nasoenteral com tubo de Dobbie e Hoffmeister ou prótese endoscópica, conforme o caso e após discussão com as equipes de radioterapia e oncologia clínica.

Emprega-se a toracotomia em tumores localizados nos segmentos esofágicos superior e médio ou em pacientes com doença avançada, mas potencialmente curável e, a via transdiafragmática para tumores de segmento esofágico inferior ou restritos a mucosa. A anastomose esofagogástrica é sempre realizada em nível cervical, com sutura manual em dois planos conforme relatado previamente e posicionamento de dreno-sentinela de Penrose.

Muitos fatores contribuem para a deiscência da anastomose esofagogástrica cervical, sendo a não observância de preceitos técnicos e isquemia oculta do tubo gástrico os mais prevalentes ${ }^{17,43,60,61}$. Portanto, o pré-requisito mais importante para a cicatrização desta anastomose é a vascularização adequada do tubo gástrico. É vital para o sucesso da anastomose que ela seja realizada sem tensão $0^{29,53,60}$, com cuidadosa aposição das camadas mucosas e com manipu- 
lação delicada dos tecidos visando manter a integridade da rica rede vascular intramural, mas isquêmica ${ }^{2,6}$ visto que o neo-esôfago confeccionado com o estômago tem vascularização tênue pela artéria e veia gastroepiplóica direita e, em alguns casos, dos vasos gástricos direitos. A correta dissecção do estômago associada ou não à manobra de Kocher permite a confecção de um tubo gástrico de dimensões capazes de atingir a região cervical, conferindo condições ótimas para anastomose segura ${ }^{29,31,60}$. A preferência pela região cervical deve-se ao fato da benignidade das fístulas neste plano anatômico ${ }^{20,21,22}$. No mesmo nível de importância, é prioritário o manejo adequado do paciente no período perioperatório, evitando a hipotensão e seu impacto negativo na perfusão sistêmica e oxigenação tecidual, sendo relevante a manutenção de adequado volume intravascular e evitando o uso de drogas vasopressoras ${ }^{22}$. O sangramento acima de $1000 \mathrm{~mL}$ também tem sido referido como associação deletéria à cicatrização da anastomose ${ }^{60}$.

Dentre os fatores responsáveis pela qualidade da anastomose, citam-se fatores intrínsecos ao procedimento, tais como anastomose realizada fora da cavidade abdominal, a ausência de camada serosa esofágica e a orientação longitudinal das fibras musculares da camada superficial do esôfago, que contribuem para a fragilidade da sutura nesta região. Dentre os fatores sistêmicos, podem-se citar desnutrição, hipotensão, hipoxemia, idade avançada, diabetes, icterícia, insuficiência respiratória ou renal e terapia neoadjuvante ${ }^{5,6,54,60}$.

Após o desenvolvimento do recurso técnico aqui relatado, observa-se a ausência de fístulas cervicais nesta série. Revisando a literatura médica recente, encontram-se taxas de deiscência entre $0,8 \%$ (série publicada em 1999 pelo Johns Hopkins Medical Institutions com 262 pacientes, na qual foi realizada esofagogastroanastomose látero-terminal em dois planos com sutura interrompida) ${ }^{28}$ e $47,6 \%$ (série publicada em 2004 pelo Departamento de Cirurgia da Faculdade de Ciências Médicas da Santa Casa de São Paulo em 60 pacientes $)^{26}$. No presente estudo, o grupo A apresentou incidência de fístula de $18,8 \%$. Registre-se que nesta série há provável viés de seleção, com tendência a resultados inferiores haja vista a participação de vários cirurgiões e residentes com distinto treinamento na confecção desta anastomose. O grupo B, que não apresentou deiscência da anastomose esofagogástrica, teve a maioria dos procedimentos realizados pelos cirurgiões-seniores deste serviço, o que corrobora a literatura quando afirma que os resultados satisfatórios de uma cirurgia de grande porte, como a esofa- gectomia, são volume dependentes ${ }^{6,9,10,49}$, e que esses bons resultados só são possíveis quando a operação é realizada em centros especializados com equipes capazes de prover a melhor experiência cirúrgica, anestésica e clínica ${ }^{14,19,22,42}$. Por outro lado, ressalte-se que é muito provável que se fossem comparadas às técnicas aqui descritas com realização somente pelos cirurgiões-seniores o resultado obtido também seria melhor nesta nova alternativa. Entretanto, cabe advertir que as taxas de deiscência de anastomose cervical da literatura podem ser sub-notificadas, visto que muitos centros não realizam exame contrastado de rotina, considerando o diagnóstico de fístula cervical apenas quando há manifestações clínicas compatíveis ${ }^{34}$.

Nesta amostra, os dois grupos analisados não diferiram estatisticamente em suas características clínicas, exceto pela idade - o grupo com idade média mais avançada apresentou taxa de fístula cervical inferior à do grupo mais jovem, fato este considerado curioso. A alternativa técnica aqui relatada apresenta como vantagem principal a facilitação da anastomose por perfeita identificação dos planos de sutura e que, juntamente com a redução da tensão na linha de sutura, foi fator relevante para a cicatrização eficaz.

Como um dos objetivos principais da operação, tanto curativa quanto paliativa, é o alívio da disfagia ${ }^{27}$ e a ocorrência de deiscência da anastomose esofagogástrica acarreta em elevado índice de estenose ${ }^{60}$, pode-se afirmar, assim como outros $^{8,12,27,34}$, que a qualidade de vida dos pacientes é diretamente influenciada pela patência de suas anastomoses. Estudo publicado em $2008^{20}$ demonstrou que em até 24 meses após a esofagectomia os pacientes não voltavam a apresentar os níveis de disfagia do pré-operatório, evidenciando o benefício da terapêutica cirúrgica no alívio sintomático.

A busca dos cirurgiões por alternativas que diminuam as complicações técnicas no pós-operatório não se deve apenas à manutenção da qualidade de vida, mas também à prevenção da mortalidade hospitalar, que em caso de intercorrências técnicas pode ser triplicada54. Curiosamente, outras séries inferem que a mortalidade a longo prazo não foi afetada pelo índice de intercorrências técnicas ${ }^{3,5,21}$.

\section{CONCLUSÃO}

A alternativa técnica proposta foi eficiente no quesito fístula por otimizar a apresentação dos planos de sutura, facilitando a confecção da anastomose, diminuindo assim as taxas desta complicação na esofagectomia. 
Fontes PRO, Kruse CK, Waechter FL, Nectoux M, Fochesato LBS, Goettert GF, Silva MF, Pereira-Lima L. Is it possible to reduce the fistula rate of the cervical esophagogastric anastomosis after oesophagectomy? ABCD Arq Bras Cir Dig. 2008;21(4):158-63

ABSTRACT - Background - The esophageal cancer is the 6th cause of cancer-related death in Brazil, and esophagectomy is the cornerstone of the treatment, not only for the curative cases, but with palliative intent as well. The cervical fistula is a very frequent complication of the procedure with a related incidence between 0,8 and 47,8\%. Aim - Prospective analysis to determine the success of a new alternative technique of cervical anastomosis. Methods - Prospective analysis of 126 patients, distributed in two groups accordingly to the cervical anastomosis technique. Group A ( $\mathrm{n}=96)$ had cervical anastomosis in two layers, the inner continuous type and outer isolated, followed by redundant tissue. Group B ( $\mathrm{n}=25)$ had a technical variation with suture done without mucosa opening at first. Results - This alternative technique for the cervical esophagogastric anastomosis presented zero fistulas. Conclusion - This alternative was efficient in decrease fistula rate because it allow best exposition and visualization of the anastomosis, facilitating the suture lines.

HEADINGS - Esophagectomy. Esophageal neoplasm.

\section{REFERÊNCIAS}

1. Akiyama H, Tsurumaru M, Kajiyama Y. Radical lymph node dissection for cancer of the thoracic esophagus. Ann Surg 1994;220(3):364-73.

2. Akiyama H, Miyazono H, Tsurumaru M, Hashimoto C, Kawamura T. Use of the Stomach as an Esophageal Substitute. Ann Surg 1978;188:606-10.

3. Ancona E, Cagol M, Epifani M, Cavalin F, Zaninotto G, Castoro C, Alfieri R, Ruol A. Surgical complications do not affect longterm survival after esophagectomy for carcinoma of the thoracic esophagus and cardia. J Am Col Surg 2006;203:661-9.

4. Ando N, Ozawa S, Kitagawa Yet al. Improvement in the results of surgical treatment of advanced squamous oesophageal carcinoma during 15 consecutive years. Ann Surg 2000; 232(2): 225-32

5. Atkins BZ, Shah AS, Hutcheson KA, Mangum JH, Pappas TN, Harpole DH, D'Amico TA. Reducing hospital morbidity and mortality following esophagectomy. Ann Thorac Surg 2004;78:1170-6.

6. Bartels H, Stein HJ, Siewert JR. Preoperative risk analysis and postoperative mortality of oesophagectomy for respectable oesophageal cancer. Br J Surg $1998 ; 85: 840-4$

7. Bartels H, Stein HJ, Siewert JR. Preoperative risk analysis and postoperative mortality of oesophagectomy for respectable oesophageal cancer. Br J Surg 1998; 85:840-4.

8. Blazeby JM, Farndon JR, Donovan J, Alderson D. A prospective longitudinal study examining the quality of life of patients with esophageal carcinoma. Cancer 2000;88:1781-7.

9. Blazeby JM. Measurement of outcome. Surg Oncol 2001; 10:127-33.

10. Blazeby JM, Conroy T, Hammarlid E, et al. Clinical and psicometric validation of an EORTC questionnaire module, the EORTC QLQ-OES18, to assess quality of life in patients with esophageal cancer. Eur J Cancer 2003; 39:1384-94.

11. Begg CB, Cramer LD, Hoskins WJ, et al. Impact of hospital volume on operative mortality for major cancer surgery. JAMA 1998; 280: 1747-51.

12. Behzadi A, Nichols FC, Cassivi SD, Deschamps C, Allen MS, Pairolero PC. Esophagogastrectomy: the influence of stapled versus hand-sewn anastomosis on outcome. J Gastroint Surg 2005:9:1031-42.

13. Cassivi SD. Leaks, strictures and necrosis: a review of anastomotic complications following esophagectomy. Semin Thorac Cardiovasc Surg 2004;16:124-32.

14. Casson AG, Van Lanschot JJ. Improving outcomes after esophagectomy: the impact of operative volume. J Surg Oncol 2005; 92:262-6.

15. Cecconello I, Zilberstein B, Domene CE, Mericoni MT, Pinotti HW. Tratamento paliativo do câncer de esôfago. Arq Bras Cir Dig 1990;5:17-20.

16. Cecconello I, Zilberstein B, Sallum RAA, Oliveira MA, Rocha JRM, Pinotti H. Câncer do esôfago: resultados do tratamento cirúrgico em 250 pacientes. Rev Col Bras Cir 1999;26:36.

17. Dewar L, Gelfand G, Finley RJ, Evans K. Factors affecting cervical anastomotic leak and stricture formation following esophagogastrectomy and gastric tube interposition. Am J Surg 1992; 163:484-9.

18. Dimick JB, Goodney PP, Orringer MB et al. Specialty training and mortality after oesophageal cancer resection. Ann Thorac Surg 2005; 80: 282-6.

19. Dimick JB, Wainess RM, Upchurch GRJr, et al. National trends in outcomes for esophageal resection. Ann Thorac Surg 2005; 79:212-6.

20. Egberts JH, Schniewind B, Bestmann B, Schafmayer C, Egberts F, Faendrich $\mathrm{F}$, Kuechler T, Tepel J. Impact of the site of anastomosis after oncologic esophagectomy on quality of life--a prospective, longitudinal outcome study. Ann Surg Oncol 2008; 15(2):566-75.

21. Ferri L, Law S, Wong KH, Kwok KF, Wong J. The influence of technical complications on postoperative outcome and survival after esophagectomy. Ann Surg Oncol 2006;13:557-64.

22. Finlayson EV, Goodney PP, Birkmeyer JD. Hospital volume and operative mortality in cancer surgery: a national study. Arch Surg 2003;138:721-5.
23. Fontes PRO, Moreira LB, Chaves AG, Varella MAS, Pereira-Lima L. O emprego de próteses como tratamento paliativo das neoplasias malignas do esôfago. Rev AMRIGS 1987;31(1):17-21

24. Fontes PRO, Nectoux M. Carcinoma de esôfago. Rev Med Sta Casa 1989;1(1):64-70.

25. Fontes PRO, Jotz GP, Jotz JCP, Moreira LB. Tunelização esofágica de 70 pacientes: pós-operatório e sobrevida. Rev Pesquisa Médica 1990;24(2):72-7.

26. Gagliardi D, Corsi PR, Frimm CE, Fava J. Câncer de esôfago - complicações pós-operatórias imediatas e letalidade hospitalar. Rev Col Bras Cir 2004; 31(1)2-9.

27. Headrick JR, Nichols FC 3rd, Miller DL, et al. High-grade esophageal dysplasia. long-term survival and quality of life after esophagectomy. Ann Thorac Surg 2002;73:1697-1702.

28. Heitmiller RF, Fischer A, Liddicoat JR. Cervical esophagogastric anastomosis: results following esophagectomy for carcinoma. Dis Esophagus 1999;12:264-9.

29. Hölscher AH, Vallböhmer D, Brabender J. The prevention and management of perioperative complications. Best Pract Res Clin Gastroenterol 2006;20(5):907-23.

30. EIlias EJ. Como estadiar o câncer de esôfago? Rev Assoc Med Bras 2007;53(4):283-92.

31. Karl RC, Schreiber R, Boulware D et al. Factors affecting morbidity, mortality and survival in patients undergoing Ivor Lewis esophagectomy. Ann Surg 2000;23:635-43.

32. Kim HK, Choi YH, Shim JH, Cho YH, Baek MJ, Sohn YH, Kin HJ. Endoscopic evaluation of the quality of the anastomosis after esophagectomy with gastric tube reconstruction. World J Surg 2008;32:2010-4.

33. Law S, Kwong DL, Kwok KF, et al. Improvement in treatment results and longterm survival of patients with esophageal cancer: impact of chemoradiation and change in treatment strategy. Ann Surg 2003; 238:339-47.

34. Lerut T, Coosemans W, Decker G, De Leyn P, Nafteux P, Van Raemdonck D. Anastomotic complications after esophagectomy. Dig Surg 2002;19:92-8.

35. Malafaia O, Dietz UA, Czeczco NG, Repka JCD, Brenner S, Engemann R, Thiede A. Estudo experimental comparativo entre técnicas de anastomoses do esôfago cervical em cães: suturas manual versus sutura mecânic. ABCD Arq Bras Cir Dig 1999;9(2);30-2.

36. Malafaia O. Experiência de 15 anos com o tratamento paliativo do câncer de esôfago através da tunelização esofágica. Rev Col Bras Cir 1986;13(5):211-5.

37. Malafaia, O. Câncer de esôfago. ABCD Arq Bras Cir Dig 1988;3;33-6.

38. Mariette C, Taillier G, Van Seuningen I et al. Factors affecting postoperative course and survival after en bloc resection for oesophageal carcinoma. Ann Thorac Surg 2004; 78: 1177-83.

39. Mathews HR, Powell DJ, McConkey CC. Effect of surgical experience on the results of resection for oesophageal carcinoma. Br J Surg 1986; 73:621-3.

40. McCulloch P, Ward J \& Tekkis PP. Mortality and morbidity in gastro-oesophageal cancer surgery: initial results of ASCOT multicenter prospective cohort study. BMJ 2003; 327: 1192-7.

41. McLarty AJ, Deschamps C, Trastek VF, Allen MS, Pairolero PC, Harmsen MS. Esophageal resection for cancer of the esophagus: long-term function and quality of life. Ann Thorac Surg 1997;63:1568-72.

42. Metzger R, Bollschweiler E, Vallbohmer D, et al. High volume centers for esophagectomy: what is the number needed to achieve low postoperative mortality? Dis Esophagus 2004;17:310-4.

43. Miller JD, Jain MK, De Gara CJ, et al. Effect of surgical experience on results of esophagectomy for esophageal carcinoma. J Surg Oncol 1997; 65:20 -21.

44. Mirra AP, Justo FA, Schneider CAR, Trippe N. Tratamento do câncer de esôfago: ensaio clínico controlado. Rev Col Bras Cir 1997;25:119-22.

45. Moura E, Sakai P, Cecconello I, Guerra P, Cenatti A, Mucenic M, Maluf Filho F, Ishioka S. Tratamento paliativo do câncer avançado do esôfago. GED 2001;19(6):215-24. 
46. Müller JM, Erasmi H, Stelzner M, Zieren U, Pichlmaier H. Surgical therapy for esophageal carcinoma. Br J Surg 1990;77:845-57.

47. Mota OM, Faintuch, J, Silva OQ, Csmpoli PMO, Machado MM, Filho JSB, Milhomem DM. Repercussões metabólico-nutricionais da reconstrução de trânsito após esofagectomia no câncer de esôfago: análise comparativa de coloplastia versus gastroplastia. ABCD Arq Bras Cir Dig 2003;16(4):193-6.

48. Orsi F, Orsi M, Carvalho G, Ginçlves R, Santos H. A fístula esôfago-gástrica cervical. Rev Col Brás Cir 2003;30:16-20.

49. Osoba D, Rodrigues G, Myles J, et al. Interpreting the significance of changes in health-related quality-of-life scores. J Clin Oncol 1998; 16:139-44.

50. Pierie JP, de Graff PW, Poen H, van der Tweel I, Obertop H. Incidence and management of benign anastomotic stricture after cervical oesophagogastrectomy. Br J Surg 1993;80:471-4.

51. Pinotti HW, Cecconello I, Oliveira MA. Transhiatal esophagectomy for esophageal cancer. Sem Surg Oncol 1998;13(4):253-8.

52. Pinto CE, Dias JD, Sá EAM, Tsunoda AT, Pinheiro RN. Tratamento cirúrgico do câncer de esôfago. Rev Bras Cancerologia 2007;53(4):425-30.

53. Rahamim J, Cham CW. Oesophagogastrectomy for carcinoma of the oesophagus and cardia. Br J Surg. 1993;80: 1305-9.

54. Rizk NP, Bach PB, Schrag D, Bains MS, Turnbull AD, Karpeh M, Brennan MF, Rusch VW. The impact of complications on outcomes after resection for esophageal and gastroesophageal junction carcinoma. J Am Coll Surg 2004; 198:42-50.

55. Rocha JRM, Cecconello I, Raimondi AM, Felix VN, Sallum RAA, Olivveira MA, Domene CE, Tacconi MRO, Gama-Rodrigues JJ. Esofagectomia transhiatal e gastroplastia no megaesôfago avançado - complicações pós-operatórias e resultados tardios. ABCD - Arq Bras Cir Dig 2002;15:9.
56. Schirmer CC, Gurski R, Castro MAA, Madruga GSP, Pedroso FL, Kruel CDP, Brentano L. Neoplasias associadas ao carcinoma epidermóide do esôfago. Rev Assoc Med Bras 1997;43(4):335-9.

57. Sutton DN, Wayman J, Griffin SM. Learning curve for oesophageal cancer surgery. Br J Surg 1998; 85:1399-1402.

58. Tomasich FDS, Valladares GCG, Demarchi VCA, Gagliardi D. Influência do tratamento neoadjuvante na morbi-mortalidade das esofagectomias. Rev Assoc Med Bras 49(3):300-5.

59. Thuler FT, Forones NM, Ferrari AP. Neoplasia avançada de esôfago - diagnóstico ainda muito tardio. Arq Gastroenterol 2006;43(3):206-11.

60. Urschel JD. Esophagogastrostomy anastomotic leaks complicating esophagectomy: a review. Am J Surg 1995;169:634-40.

61. Urschel JD, Blewett CJ, Bennet WF, Miller JD, Young JEM. Handsewn or stapled esophagogastric anastomosis after esophagectomy for cancer: metaanalysis of randomized controlled trials. Dis Esophagus 2001;14:212-7.

62. Whooley BP, Law S, Murthy SC, Alexandrou A, Wong J. Analysis of reduced death and complication rates after esophageal resection. Ann Surg 2001; 23(3):338-44.

63. Young MM, Deschamps C, Trastek VF, et al. Esophageal reconstruction for benign disease: early morbidity, mortality, and functional results. Ann Thorac Surg 2000;70:1651-5.

Fonte de financiamento: não há Conflito de interesse: não há Recebido para publicação: 18/05/2008 Aceito para publicação: 21/08/200 